\title{
Pulmonary Embolism - a Thrombo- Inflammatory Complication of a Viral Infection. The Paradigm Shift in the COVID Era
}

\author{
Theodora Benedek \\ "George Emil Palade" University of Medicine, Pharmacy, Sciences and Technology, Târgu Mureș, Romania
}

Pulmonary embolism (PE) is a major cardiovascular emergency that has been traditionally associated with increased blood coagulability and prolonged immobilization. The embolization of a large thrombus from the deep venous system, developed usually after major pelvic surgery or hip fracture, was one of the most frequent conditions leading to massive $\mathrm{PE}$, a life-threatening condition. ${ }^{1}$

Increased blood coagulability has also been involved in the etiology of PE. A particular case is represented by the congenital deficit of anticoagulant proteins $\mathrm{S}$ and $\mathrm{C}$, two plasma proteins that act as natural anticoagulants. In a recent prospective family cohort study, family members exhibiting an inherited deficiency of natural anticoagulants presented a five-times higher risk of vascular thrombosis than the control population. ${ }^{2}$ This is of particular relevance in cases where none of the clinical and paraclinical examinations are able to identify the source of embolism. In these cases, a potential etiology could be represented by increased blood coagulability resulting from the deficit of these proteins. From a clinical point of view, this means that in a patient with $\mathrm{PE}$ and no sign of deep vein thrombosis, serum levels of protein $\mathrm{C}$ and protein $\mathrm{S}$ should be assessed in order to identify a systemic condition that may predispose to PE.

In spite of the many efforts oriented towards the diagnosis of potential etiologies for cases of PE with unidentified source, there is no clear association described so far between a viral infection and PE. A virus able to increase blood coagulability may be responsible for catastrophic events in the entire cardiovascular system.
Such a virus seems to be the new SARS-CoV-2, a virus that has emerged in Wuhan (China) at the end of 2019 and causes the novel COVID-19 disease. A huge amount of data has been published on the new coronavirus infection in the latest months worldwide, in the attempt to identify the pathophysiologic mechanisms involved in the dramatic evolution of critical COVID-19 cases. However, given the relatively short period of time since the outbreak started, it is quite difficult to generate scientific conclusions based on studies lacking an appropriate period of follow-up. Still, it has been reported and generally accepted that COVID-19 is responsible for a sudden increase in systemic inflammation, which may favor thrombosis in the small vessels, including in the pulmonary vasculature. This may explain the relatively high incidence of PE in patients with COVID-19 presenting clinical deterioration. In a recent study from Italy, PE was identified in $57 \%$ of patients with severe COVID-19 pneumonia, despite ongoing anticoagulant therapy. ${ }^{3}$

In a recent meta-analysis of 102 studies, venous thromboembolism was identified in $14.7 \%$ of COVID-19 patients, and the prevalence of PE was $7.8 \%$. Interestingly, the incidence of venous thrombosis was higher in patients systematically screened for deep vein thrombosis than in those who were tested only based on symptoms. ${ }^{4}$

These observations indicate a paradigm shift in the definition of PE during the recent COVID era. PE is no longer a disease associated with prolonged immobilization, major surgery or blood coagulability; it is also a thrombo- 
inflammatory complication of a viral infection. As opposed to patients with non-COVID PE, those with COVID and PE have no history of recent immobilization or major surgery.

It is largely accepted nowadays that a CT scan, investigation that has become routine for patients with suspicion of PE, may screen the entire pulmonary bed not only for the signs of pulmonary infection but also for the presence of PE. At the same time, a PCR test for COVID-19 should be performed in all cases with $\mathrm{PE}$, since the PE may be caused by the SARS-CoV-2 infection.

However, in light of the currently available data, it is difficult to define whether the altered clinical status of a patient with severe COVID-19 infection and PE is the result of the systemic infection with exacerbated inflammation, which also leads to $\mathrm{PE}$, or rather the result of $\mathrm{PE}$, which deteriorates the fragile equilibrium of the critically ill patient. One thing is clear: today, we are facing an increasing number of patients with PE caused by COVID-19, with a different clinical presentation than the one we knew.

The paradigm shift of PE pathophysiology in the COVID-19 era may also raise a particular concern regarding the future of COVID-19 survivors, since once triggered, the inflammatory cascade may continue to favor endothelial dysfunction and thrombosis on long term. This may favor the development of PE even after the patient has healed from COVID-19.
All these data and hypothesis remain speculative until large randomized trials will confirm them. Even if by that moment the world will recover from the COVID-19 disease, evidence driven from these studies will remain extremely valuable for the future. Who knows what the future will bring...???

\section{CONFLICT OF INTEREST}

Nothing to declare.

\section{REFERENCES}

1. Capilna M, Moldovan B, Szabo B. Pelvic exenteration our initial experience in 15 cases. European Journal of Gynecological Oncology. 2015;36:142-145.

2. Tormene D, Noventa F, Campello E, et al. The risk of arterial thrombosis in carriers of natural anticoagulation inhibitors: a prospective family cohort study. Intern Emerg Med. 2021. doi: 10.1007/s11739-021-02656-5. [Epub ahead of print]

3. Valle $\mathrm{C}$, Bonaffini PA, Corso MD, et al. Association between pulmonary embolism and COVID-19 severe pneumonia: Experience from two centers in the core of the infection Italian peak. Eur J Radiol. 2021;137:109613. doi: 10.1016/j. ejrad.2021.109613. [Epub ahead of print]

4. Tan BK, Mainbourg S, Friggeri A, et al. Arterial and venous thromboembolism in COVID-19: a study-level metaanalysis. Thorax. 2021;thoraxjnl-2020-215383. doi: 10.1136/ thoraxjnl-2020-215383. [Epub ahead of print] 